\title{
Fog-LAEEBA: Fog-assisted Link aware and energy efficient protocol for wireless body area network
}

\author{
Kifayat ULLAH \\ Department of Computer Science, \\ CECOS University of IT and Emerging \\ Sciences, Peshawar, Pakistan \\ email: kifayat@cecos.edu.pk
}

\author{
Haris KHAN \\ Department of Computer Science, \\ CECOS University of IT and Emerging \\ Sciences, Peshawar, Pakistan \\ email: hariskhan5848@gmail.com
}

\begin{abstract}
The integration of Wireless Sensor Networks (WSN) and cloud computing brings several advantages. However, one of the main problems with the existing cloud solutions is the latency involved in accessing, storing, and processing data. This limits the use of cloud computing for various types of applications (for instance, patient health monitoring) that require real-time access and processing of data. To address the latency problem, we proposed a fog-assisted Link Aware and Energy Efficient Protocol for Wireless Body Area Networks (Fog-LAEEBA). The proposed solution is based on the already developed state-of-the-art protocol called LAEEBA. We implement, test, evaluate and compare the results of Fog-LAEEBA in terms of stability period, end-to-end delay, throughput, residual energy, and path-loss. For the stability period all nodes in the LAEEBA protocol die after 7445 rounds, while in our case the last node dies after 9000 rounds. For the same number of rounds, the end-to-end delay is 2 seconds for LAEEBA and 1.25 seconds for FogLAEEBA. In terms of throughput, our proposed solution increases the number of packets received by the sink node from 1.5 packets to 1.8 packets. The residual energy of the nodes in Fog-LAEEBA is also less than
\end{abstract}

Computing Classification System 1998: G.2.2

Mathematics Subject Classification 2010: $68 \mathrm{R} 15$

Key words and phrases: wireless sensor network, wireless body area network, fog computing, fog-laeeba, energy efficiency 
the LAEEBA protocol. Finally, our proposed solution improves the path loss by 24 percent.

\section{Introduction}

The advancement in emerging technologies is reshaping our world and living styles. Some of these emerging technologies are Wireless Sensor Network (WSN), Wireless Body Area Network (WBAN), and cloud computing. A large number of cloud-based solutions have been developed. One of the major drawbacks of cloud computing is the delay involved in accessing and processing data. This delay can significantly influence network performance [1]. Cloud computing does not fulfill the real-time requirements of delay-sensitive applications [14]. To address this limitation of cloud computing the idea of fog computing was proposed. Fog computing is a new paradigm shift in providing cloud services at the edge of the network. It provides tools for managing, distributing, and securing resources and facilities across networks and between devices that exist at the edge of the network [4].

WBAN is a special type of WSN in which several small lightweight wireless sensors are placed or implanted in human bodies for health monitoring [8]. The main objective is to monitor, collect, and report medical data from the patient body $[12,3]$. Different applications have been proposed using WBAN. For example, patient monitoring, sudden fall of a patient, informing an emergency response unit, call, sudden falls of patients, and providing guidance to the patients, etc.

Current solutions for monitoring patient health focuses on the collection of medical data and sending it towards a centralized server for making it available to the medical staff. One such solution is the so-called Link Aware and Energy Efficient Scheme for Body Area Networks (LAEEBA). It is a well-known, reliable, and efficient protocol designed for WBAN. The design of LAEEBA is based on cloud architecture. The major issue with the LAEEBA protocol is the delay involved in the data processing, which is not tolerable in the case of health-related applications [10].

We extend the operations of the LAEEBA protocol to fog computing. In this regard, we proposed the design, and implementation of fog assisted efficient solution called Fog-LAEEBA. The main focus is to address the problem of delay involved in the LAEEBA protocol. On one side, this will help in storing, managing, computing, and analyzing the sensory data. On the other side, it will minimize the delay involved in the LAEEBA protocol. In the proposed 
solution, we deployed different sensors on the human body to monitor the patient's health-related data. Such data includes blood pressure, pulse rate, and temperate, etc. This information will be transferred to a sink node, placed on the body. The sink node will then send the data to the fog node.

The general objective of this research is to extend the operations of the LAEEBA protocol to support the fog computing paradigm. To this end, the more specific objectives are:

- To design a fog-assisted solution for patient health monitoring;

- To implement, test, and evaluate the performance of the proposed solution in terms of stability period, delay, throughput, path-loss, and energy utilization;

- To compare the result of the proposed solution with the LAEEBA protocol.

\section{Literature review}

In the literature different algorithms, and protocols have been proposed for WBAN using cloud and fog computing. These solutions include different applications for real-time health monitoring, improving network performance, enhancing energy utilization, and minimizing the delay involved in processing. In this section, we provide a literature review of the existing solutions.

A detailed overview of WBAN is provided in [3]. This work highlights both the medical and non-medical applications. It also specifies the requirements for different types of sensors, which are used in WBAN. Furthermore, this work outlines the communication technologies, like, Global System for Mobile communication (GSM), Wireless Personal Area Network (WPAN), WiFi, cellular networks, ZigBee, and Bluetooth for WBAN.

As the sensor nodes in WBAN are battery-powered, hence, it is important to efficiently utilize the energy of these nodes. To increase the network performance of WBAN, an Identification Key Scheme (IKS) was proposed in [2]. The IKS improves the performance of WBAN in terms of minimizing the packet loss, and end-to-end delay. The authors used the OMNET++ simulator to implement and evaluate the performance of IKS. Our proposed scheme also considered these evaluation metrics, however, we implement our scenario in the presence of a fog unit.

An analytical model, to improve the network performance of healthcare applications, is proposed in [13]. The proposed solution recommends the use 
of fuzzy logic and fog computing to reduce the latency involved in the WBAN. The proposed solution was implemented in the iFogSim simulator. The authors only consider network latency metric for the evaluation of the proposed model.

A Long Range (LoRa) based, fog-assisted health monitoring application is proposed in [7]. The proposed work highlight the importance of a health monitoring system. To address the need for a proper health system in practice, the authors emphasize the use of fog computing, IoT, and LoRa communication technologies. They also proposed an architecture for the proposed monitoring system. Finally, the authors developed a Raspberry Pi-based test-bed to perform their experiments. However, they focus on the communication aspect (e.g., RSS, and SNR) of the LoRa. One of the main drawbacks of the proposed solution is the placement of fog nodes. In this work, the fog nodes are placed health centers and hospitals, which are far away from the patient homes.

As the aging population of the world is increasing. There is a social need to address the health issues of this large community. To address this problem, a fog computing-based elderly health monitoring system is proposed in [5]. The proposed system utilizes the Mysignals HW kit for measuring health parameters. They also developed a mobile application to analyze the collected information.

A fog computing and K-Nearest Neighbor (KNN) based health monitoring system for heart patient monitoring is proposed in [11]. This work makes use of fog computing to minimize the delay problem and also enhance the accessibility of the system. The main focus of this work is to monitor the heart disease patient and alert the doctor, in case of an emergency. The authors implement their solution by using an Arduino board, Raspberry pi device, and heart rate sensor module. They evaluated the performance in terms of accuracy only.

The authors in [9] proposed a scheme called AnyCasting In Dual Sink (ACIDS) for enhancing the performance of WBAN. This work focuses on enhancing the performance of the WBAN in terms of throughput and stability period. Like our proposed solution, they compared the results with the LAEEBA protocol. This work does not give attention to energy efficiency, which is important to consider to evaluate the performance of WBAN. Furthermore, their solution does not consider the use of the fog computing paradigm.

The major inspiration behind our proposed solution is the protocol presented in [10]. This protocol is called Link Aware and Energy Efficient Scheme for BAN (LAEEBA). The LAEEBA is a well-recognized, reliable, and efficient routing protocol designed for WBAN. The LAEEBA protocol tries to improve the energy efficiency of the network by maximizing the network throughput and minimizing delay. For efficient path selection, it utilizes the number of hops 
while for energy consumption a cost function is calculated. The idea of cooperative Link-Aware and Energy Efficient protocol for WBAN (Co-LAEEBA) is provided in [?]. This work focuses on the impact of single-hop and multihop communication techniques. It also highlights the importance of cooperation among sensor nodes. The main conclusion was that such cooperation can maximize the throughput of a network.

\section{$3 \quad$ Research methodology}

The main objective of this research work is to propose a fog-assisted protocol for WBAN. To achieve this goal, we followed a step-by-step research methodology. This section presents the most important steps of our methodology.

\subsection{Conduct literature survey}

To conduct this research, we performed a detailed literature review by consulting standard libraries, e.g., Google Scholar, IEEE, ACM, SpringerLink, and ScienceDirect, etc. We studied different approaches for the provision of health services using WBAN. This study enables us to identify the main limitations of the existing approaches and identify a research gap.

\subsection{Simulation tools}

For WBAN and fog computing, performing real-world experiments is a costly, laborious, and time-consuming task. Similarly, it is difficult to repeat the same experiments for different parameters. An alternative solution is to perform simulations. Simulation tools are used to implement, test, validate, compare, and evaluate the performance of the proposed solution. For this purpose, a wide range of simulation tools, ranging from open source to proprietary solutions, are available. However, the selection of the most appropriate simulation tools is an important step in any research. We used a well-known simulation tool called MATLAB. It is a matured and popular platform for scientific computing and is widely used for conducting various simulation-based studies. MATLAB has a rich set of modules that allows the user to perform different tasks.

\subsection{Simulation scenario}

After the selection of simulation tool, an important step to perform any simulation-based experiment is to select a suitable scenario and set up the 
simulation environment. This step is also valuable for achieving reliable simulation results. In our proposed solution, we deployed different sensor nodes on the human body. In addition, we also placed a sink node. Fig. 1 shows the placement of these sensor nodes. All the sensor nodes are homogeneous having the same computational capabilities. These sensor nodes are responsible for sensing body temperature, heart rate, and oxygen level, etc. On the other side, the sink node forwards the data collected from these sensor nodes toward the fog node for further processing.

\subsection{Simulation parameters}

To evaluate the performance of the proposed solution, it is important to define a set of parameters and metrics. The parameters that we choose for our experiments are stability period, end-to-end delay, throughput, residual energy, and path loss. These parameters are important for WBAN. In addition, the selection of this standard set of parameters would help other researchers to compare their solution with our approach.

\subsection{Performance evaluation}

To evaluate the performance of our proposed system, we performed different experiments. We studied how the selected parameters influence the performance of the proposed solution. Furthermore, we also compared the result with the LAEEBA protocol.

\subsection{Results analysis}

The last step of our proposed research methodology is to analyze the collected results of the experiments and draw some useful conclusions. We discuss our results in more detail in section 5 .

\section{Fog-laeeba}

In this section we present the details about our proposed solution. Fog-LAEEBA is an extension to the LAEEBA protocol. It addresses the limitations, e.g., delay, energy consumption, throughput, and path-loss of the LAEEBA protocol. The design of our protocol is based on fog computing paradigm. Compared to the cloud computing, the fog node is much closer to the sensor nodes and 
hence is considered to be an efficient solution, especially for health related applications.

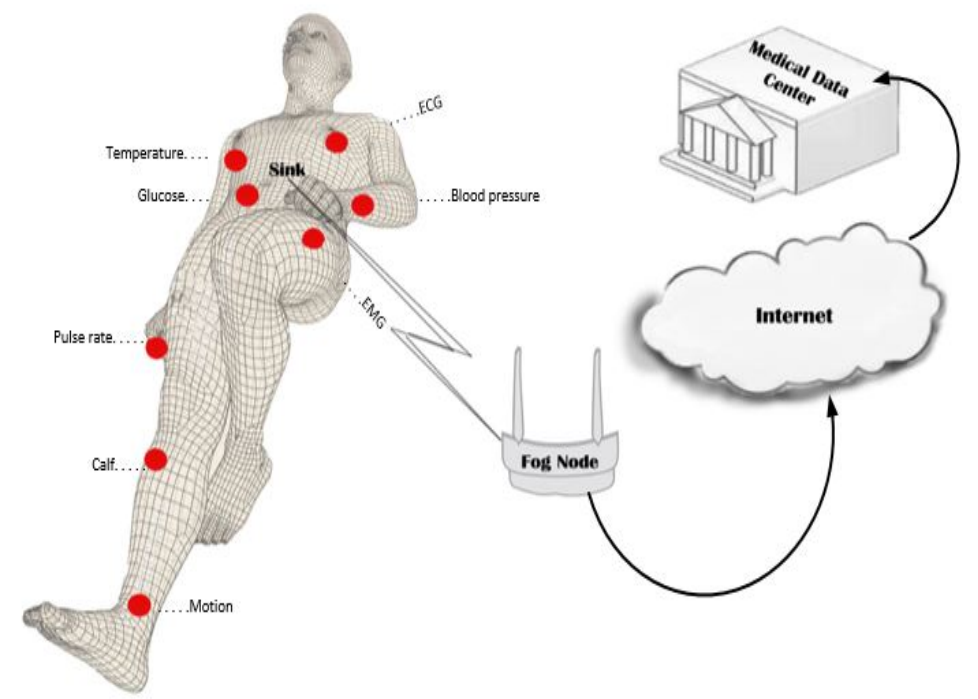

Figure 1: Proposed system model.

\subsection{System model}

In our proposed model we deployed eight sensors and one sink node. All nodes have equivalent power and computation abilities. Fig. 1 shows the placement of various sensors on the body. Each node is responsible for sensing different health-related parameters. From the sink node, the collected data is transferred to the fog Node. The flowchart of our system is also depicted in Fig. 2.

To implement our proposed solution, we used MATLAB. We perform extensive simulation experiments to evaluate and compare the performance. Table 1 indicates the various parameters used for experiments. 


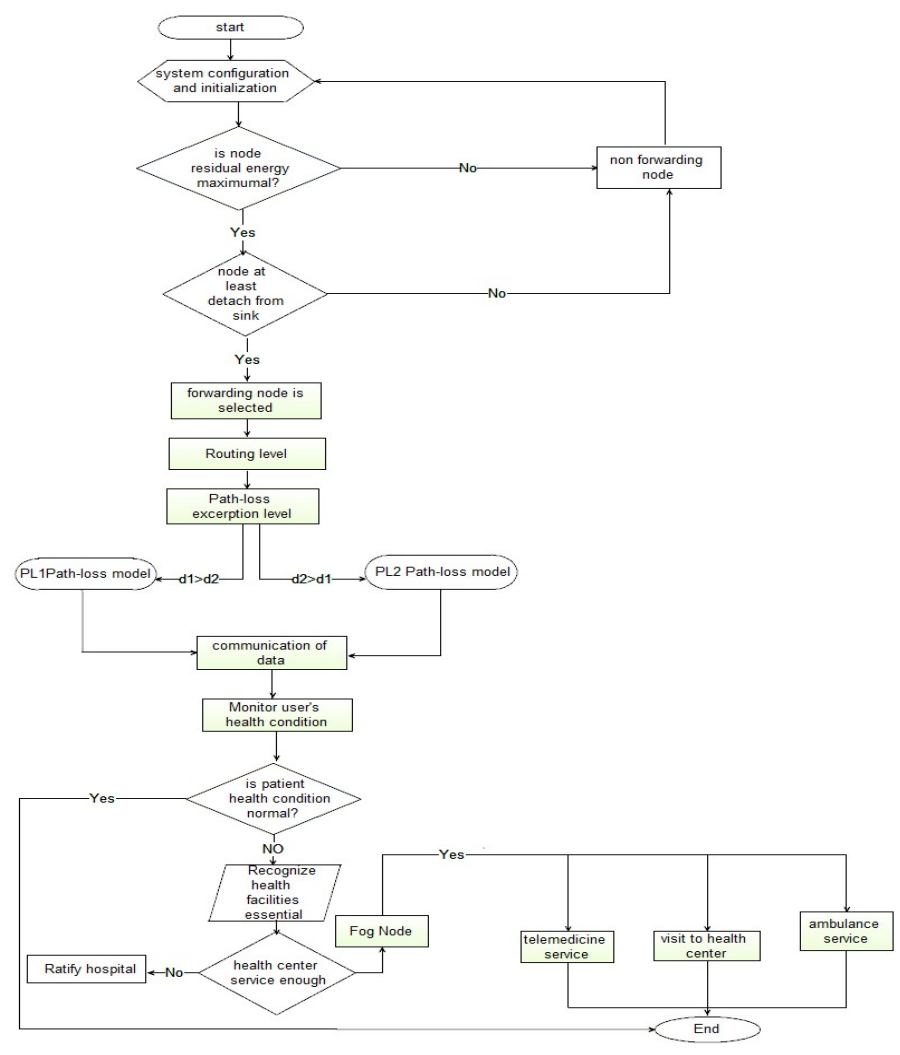

Figure 2: Flow diagram of proposed solution.

\section{Results and discussion}

This section presents the results of our simulation experiments. We also provide a comparison of the results with the existing LAEEBA protocol. It is important to note that we repeat each set of experiments five times.

\subsection{Stability period}

The first metric that we consider to evaluate the performance of Fog-LAEEBA is the stability period. The stability period is the time in which all the nodes in a network stay alive. It is considered to be the key performance evaluation metric. We present the obtained results of the stability metric in Fig. 3. The results reveal that in the LAEEBA protocol the first node dies at around 2147, while in our protocol the first node dies after 4437 rounds. After round number 


\begin{tabular}{|l||l|}
\hline Parameter & Value \\
\hline \hline Number of nodes & 8 \\
Position of nodes & On human body \\
Mobility model & First order radio Model \\
Size of packet & $1000 \mathrm{bits}$ \\
Initial Energy & $0.4 \mathrm{~J}$ \\
Eamp & $0.0013 \mathrm{PJ} / \mathrm{bit} / \mathrm{m}^{4}$ \\
Eelec & $50 \mathrm{nj} / \mathrm{bit}$ \\
Efs & $100 \mathrm{pj} / \mathrm{bit} / / \mathrm{m}^{2}$ \\
Eda & $5 \mathrm{NJ} / \mathrm{bit}$ \\
Emp & $1.97 \mathrm{NJ} / \mathrm{bit}$ \\
Initial Energy & $0.5 \mathrm{~J}$ \\
Frequency & $2.4 \mathrm{GHz}$ \\
Wavelength & $0.125 \mathrm{~m}$ \\
Standard & IEEE 802.15 .6 \\
\hline
\end{tabular}

Table 1: Simulation parameters and their values.

7445, all nodes in the LAEEBA protocol died, whereas in our proposed solution the last node dies after round number 9000. From the result, it is clear that our proposed solution performed better than the LAEEBA protocol. We can conclude that the stability period of our protocol is high, which will enhance the lifetime of the network.

\subsection{End-to-end delay}

An important parameter to consider in performance evaluation is an end-toend delay. It is the time taken by a packet to reach the destination. One of the main goals of WBAN is to decrease the end-to-end delay. We evaluate the performance of the Fog-LAEEBA with the objectives to minimize this delay.

Our simulation results are shown in Fig. 4. The results demonstrate that in terms of end-to-end delay our solution performs better as compared to LAEEBA Protocol. It can be observed that in our case, the end-to-end delay is high (i.e., 2.243 milliseconds) during the early stages. However, after some cycles, this delay is minimized. 


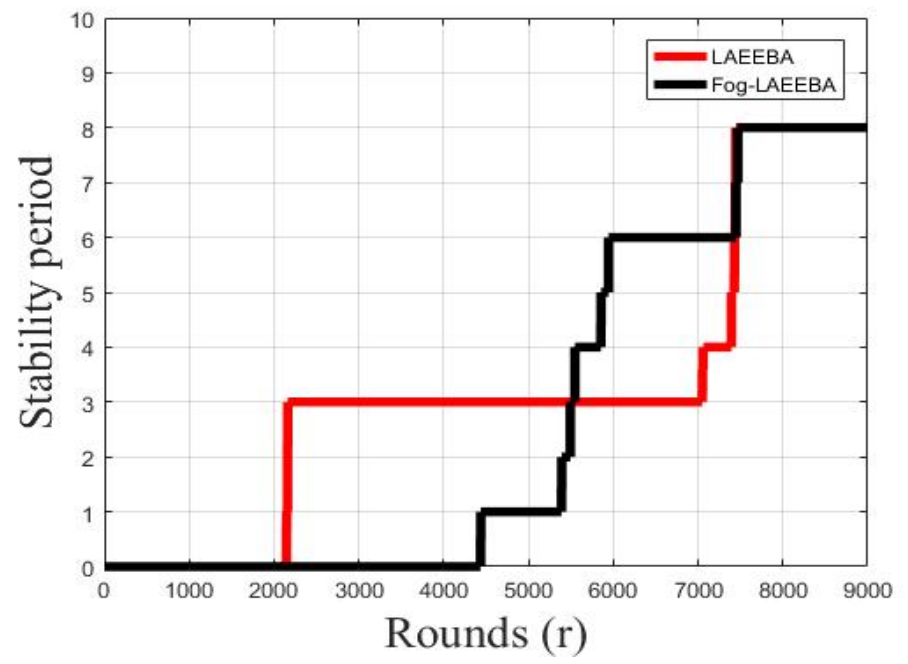

Figure 3: Stability period.

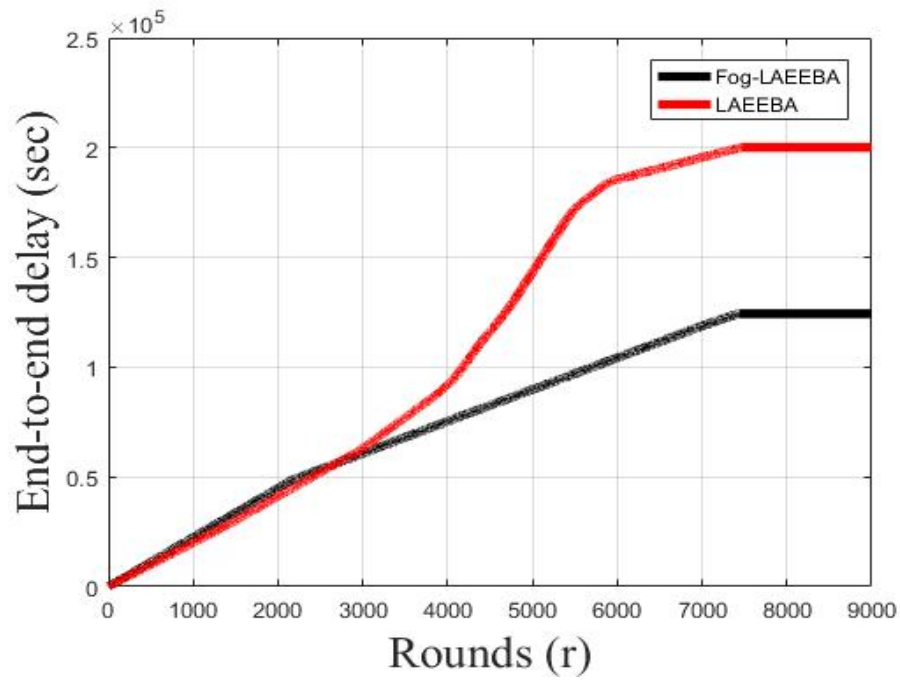

Figure 4: End-to-end delay.

\subsection{Throughput}

Throughput means the number of packets being transferred from source to destination in unit time. It is also an important factor to be considered for 
performance evaluation. The design goal of any solution should be to improve network performance by maximizing throughput. We also considered throughput for evaluating the performance of Fog-LAEEBA.

The results collected are shown in Fig. 5. At the initial stages of the simulations, the performance of both protocols is the same. However, at later stages, our proposed solution achieved higher throughput as compared to the LAEEBA protocol. In the case of the LAEEBA protocol, about 1.5 packets (on average) were received by the sink node. However, in the case of FogLAEEBA, around 1.8 packets were successfully received. From the results, we concluded that Fog-LAEEBA improved the performance by about 13 percent.

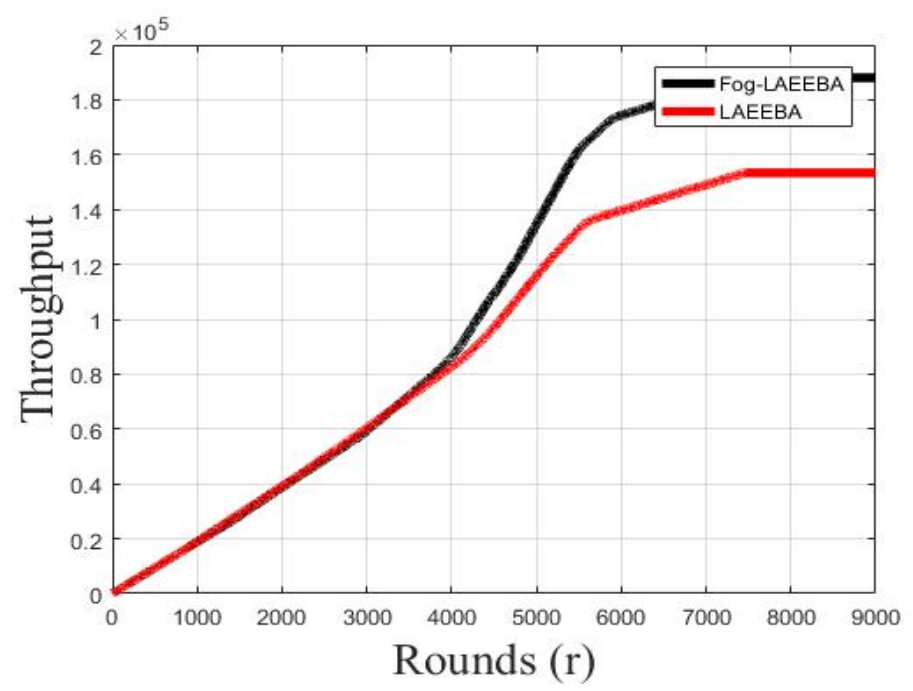

Figure 5: Throughput.

\subsection{Residual energy}

As the sensor nodes rely on batteries, hence, energy is the main concern in such types of networks. The term residual energy means the current energy of a sensor node, after performing basic operations,e .g., sending or receiving data packets. To perform the experiments, we kept the initial energy of sensor nodes to four joules. The obtained simulation results are shown in Fig. 6. From the results, it is clear that our proposed solution consumes less energy at the start of the network operation. Up to round number 4000, the residual energy of our proposed solution is less than that of the LAEEBA protocol. However, 
after that point, there is a slight variation in the energy consumption by both solutions.

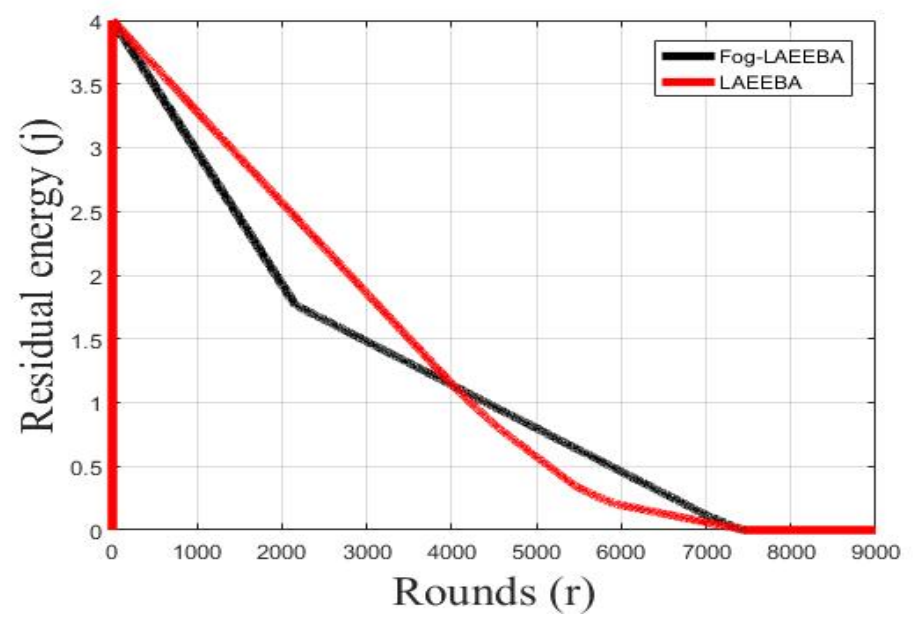

Figure 6: Residual energy.

\subsection{Path-loss}

Path loss is the reduction in the power of an electromagnetic wave. Several factors can affect the quality of the transmitted signal. Usually, in WBAN, the sensors are placed on or implanted inside the human body. Hence losses between these devices could reduce the performance of health monitoring activities. We performed different experiments to evaluate the performance of our proposed solution in terms of path-loss. The results of path-lose experiments are shown in Fig. 7. From the result, it is clear that the proposed solution reduces the path loss at various rounds. Only between 2150 and 3900, the path loss in our protocol is high. Overall, our proposed solution achieves 24 percent improvements over the LAEEBA protocol by reducing the average path loss from $316.01 \mathrm{~dB}$ to $239.95 \mathrm{~dB}$.

\section{Conclusion and future work}

WBAN and fog computing will pave the way for the deployment of several applications. One such application area is the provision of health-related services. Although the integration of WBAN and cloud computing brings several 


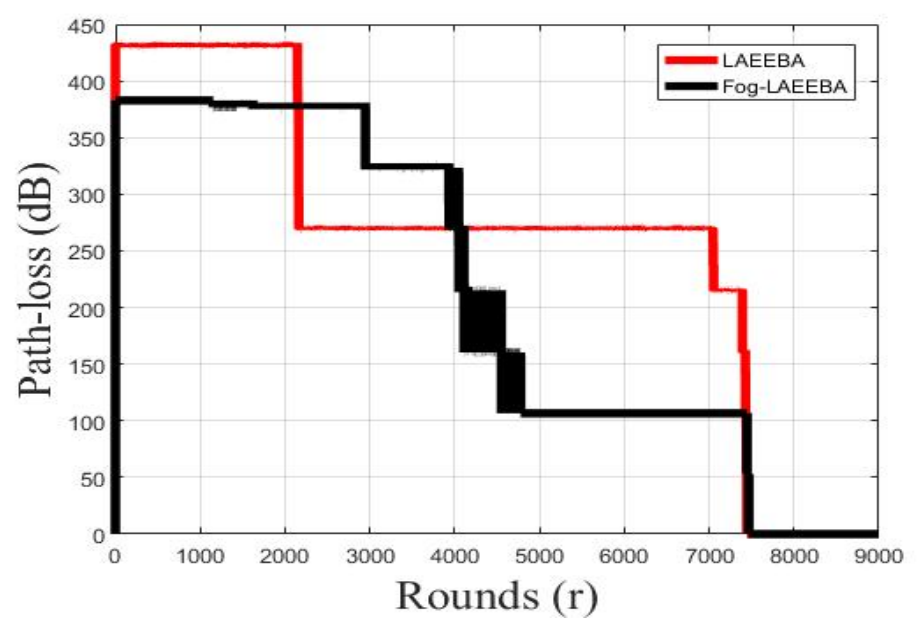

Figure 7: Path-loss.

advantages, one of the main problems with the existing cloud-based solutions is the latency involved in accessing, storing, and processing data. This limits the use of cloud computing for applications that require real-time access and processing of data. To address this challenge, we proposed Fog-LAEEBA, which follows the fog computing paradigm. Instead of sending the data to the cloud for processing, which involved delay and complexities, the sensory data would be processed locally on a fog unit. We implement, test, and evaluate the performance of Fog-LAEEBA in a simulation environment. For this purpose, we used a MATLAB simulator. Different parameters were used to evaluate the performance of our proposed solution, such as stability period, end-toend delay, and throughput, etc. From the simulation results, we conclude that Fog-LAEEBA performs better in several ways. Fog-LAEEBA minimizes the end-to-end delay, involved in the LAEEBA protocol. In terms of energy efficiency, our proposed solution enhances the lifetime of the network. Moreover, we observed that Fog-LAEEBA brings a 24 percent improvement to the existing LAEEBA protocol by reducing the average path-loss from $316.01 \mathrm{~dB}$ to $239.95 \mathrm{~dB}$.

In the future, we plan to test and deploy Fog-LAEEBA in real-world scenarios. Instead of using MATLAB simulations, we can deploy several medical sensors on the human body. Furthermore, we can also compare the results of our simulations with the results collected from real-world scenarios. This will help us to understand the gap between simulation and practical implementa- 
tion setup. Security, privacy, safety, and trust cannot be eliminated from any research work. Another future direction would be the consideration of these aspects for safe and secure operations of the Fog-LAEEBA.

Finally, we will enhance the performance of the proposed solution by taking into consideration several other parameters, e.g., sending the collected information to a nearby hospital, or medical response teams.

\section{References}

[1] A. A. Mutlag, M. K. A. Ghani, N. Arunkumar, M. A. Mohammed, O. Mohd, Enabling technologies for fog computing in healthcare IoT systems, Future Generation Computer Systems 90, (2019) 62-78. $\Rightarrow 181$

[2] A. Israa, A. Haider, S. Shihab, S. Siti, Identification key scheme to enhance network performance in wireless body area network, Periodicals of Engineering and Natural Sciences (PEN) 7, 2 (2019) 895-906. $\Rightarrow 182$

[3] A. Md. Taslim, H. M. Haque, A.K.M Fazlul, Wireless Body Area Network: An Overview and Various Applications, Journal of Computer and Communications 5, (2017) 53-64. $\Rightarrow 181,182$

[4] B. Flavio, M. Rodolfo, Z. Jiang, A. Sateesh, Fog Computing and Its Role in the Internet of Things, Proceedings of the First Edition of the MCC Workshop on Mobile Cloud Computing, Helsinki, Finland, 2012, pp. 13-16. $\Rightarrow 181$

[5] E. Moghadas, J. Rezazadeh, R. Farahbakhsh, An IoT patient monitoring based on fog computing and data mining: Cardiac arrhythmia usecase, Internet of Things 11, (2020) 2542-6605. $\Rightarrow 183$

[6] H. B. Hassen, W. Dghais, B. Hamdi, An E-health system for monitoring elderly health based on Internet of Things and Fog computing, Health Information Science and Systems 7, 1 (2019) 1-9. $\Rightarrow 183$

[7] J. Kharel, H. T. Reda, S. Y. Shin, Fog Computing-Based Smart Health Monitoring System Deploying LoRa Wireless Communication, IETE Technical Review 36, 1 (2019) 69-82. $\Rightarrow 183$

[8] K. Rani, N. Parma, Performance comparison of various routing protocols in WSN and WBAN, 2016 International Conference on Computing, Communication and Automation (ICCCA), Greater Noida, India, 2016, pp. 427-431. $\Rightarrow 181$

[9] M. R. Baig, N. Ullah, F. Hadi, S. Ahmed, A. Hanan, I. Ahmed, AnyCasting In Dual Sink Approach (ACIDS) for WBASNs, International Journal of Advanced Computer Science and Applications 8, 3 (2017) 257-263. $\Rightarrow 183$

[10] S. Ahmed, N. Javaid, M. Akbar, A. Iqbal, Z. A. Khan, U. Qasim, LAEEBA: Link Aware and Energy Efficient Scheme for Body Area Networks, 2014 IEEE 28th International Conference on Advanced Information Networking and Applications, Victoria, BC, Canada, 2014, pp. 435-440. $\Rightarrow 181,183$

[11] S. Ahmed, N. Javaid, S. Yousaf, A. Ahmad, M.M. Sandhu, M. Imran, Z.A. Khan, N. Alrajeh, Co-LAEEBA: Cooperative link aware and energy efficient protocol 
for wireless body area networks, Computers in Human Behavior 51, B (2019) $1205-1215 . \Rightarrow 183$

[12] S. Ahmed, N. Sadiq, K. Sadiq, N. Javaid, M. A. Taqi, Node Density Analysis for WBAN Schemes in Terms of Stability and Throughput, Recent Trends and Advances in Wireless and IoT-enabled Networks, Springer, Cham, 2019. $\Rightarrow 181$

[13] S. Saurabh, H. M. Fadzil, J. L. Tang, A. Azlan, K. M. Khalid, 3-Tier Architecture for Network Latency Reduction in Healthcare Internet-of-Things Using Fog Computing and Machine Learning, Proceedings of the 2019 8th International Conference on Software and Computer Applications, VPenang, Malaysia, 2019, pp. $522-528 . \Rightarrow 182$

[14] V. Prabal, S. Sandeep, Fog Assisted-IoT Enabled Patient Health Monitoring in Smart Homes, IEEE Internet of Things Journal 5, 3 (2018) 1789-1796. $\Rightarrow 181$

Received: April 24, 2021 • Revised: June 1, 2021 